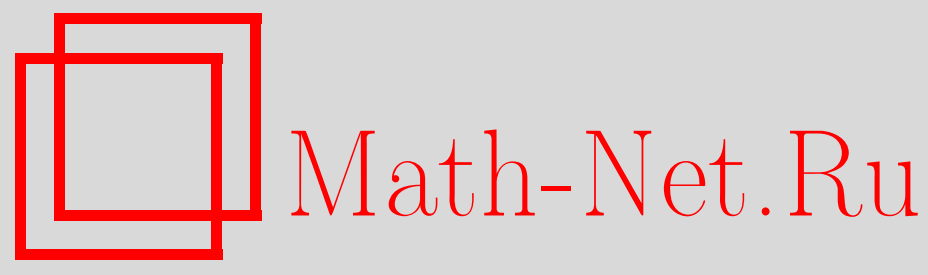

В. В. Асеев, А. В. Тетенов, А. П. Максимова, Обобщенная метрика Помпейю в проблеме изометрии гиперпространств, Матем. заметки, 2005, том 78, выпуск 2, 163-170

DOI: https://doi.org/10.4213/mzm2573

Использование Общероссийского математического портала Math-Net.Ru подразумевает, что вы прочитали и согласны с пользовательским соглашением http://www.mathnet.ru/rus/agreement

Параметры загрузки:

IP : 3.82 .47 .9

26 апреля 2023 г., 15:10:20

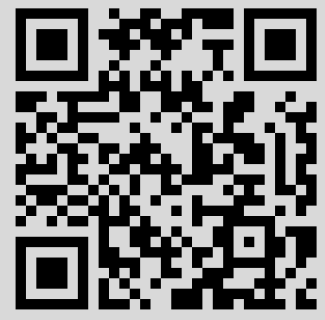




\title{
ОБОБЩЕННАЯ МЕТРИКА ПОМПЕЙЮ \\ В ПРОБЛЕМЕ ИЗОМЕТРИИ ГИПЕРПРОСТРАНСТВ
}

\author{
В. В. Асеев, А. В. Тетенов, А. П. Максимова
}

Для компактного множества на прямой рассматриваются изометрии его гиперпространства, наделенного обобщенной метрикой Помпейю. Доказывается, что любая такая изометрия порождена изометрией базового пространства.

Библиография: 14 названий.

1. Введение. Ковариантный функтор ехр в категории $\mathscr{T} о р$ сопоставляет топологическому пространству $X$ его гиперпространство $\exp X$ - семейство всех непустых компактных подмножеств, наделенное әкспоненииальной топологией $[1$, т. 1, гл. $1, \S 17$, c. 168] (синонимы: топология Вьеториса [2, гл. 2, п. 2.7.20, с. 192] и [3], конечная топология [4], топология Вьеториса-Хаусдорфа; в метрическом случае - топология Хаусдорфа). Для гиперпространств используют и обозначение $2^{X}$ (см. [1], [4], [2]).

Очевиден отрицательньй ответ на вопрос о том, будет ли любой гомеоморфизм $F$ : $\exp X \rightarrow \exp Y$ әлементарным, т.е. $F=\exp f$ для некоторого гомеоморфизма $f: X \rightarrow Y$ (пространства $X$ и $Y$ могут даже не быть гомеоморфньпи друг другу [4, с. 170], [1, т. 2, гл. $4, \S 42$, с. 53]); простой контрпример - это $n$-точечное дискретное пространство $X$, для групшы автоморфизмов которого card $\operatorname{Aut}(X)=n !<\operatorname{card} \operatorname{Aut}(\exp X)=\left(2^{n}-1\right)$ !.

Ограничение функтора ехр на подкатегорию $\mathscr{M}$ etr связано с выбором способа метризации гиперпространства, согласованного с экспоненциальной топологией, при котором каноническое вложение $x \rightarrow\{x\}$ является изометрическим. В случае компактных метрических пространств $(X, \rho)$ таковой является метрика Хаусдорфа dist на $\exp X[1$, т. 1 , гл. $2 . \S 21$, с. 223 ; т. 2 , гл. $4 . \oint 42 . \mathrm{II}$, с. 54$]$ :

$$
\operatorname{dist}(A, B)=\max \left\{\sup _{x \in A} \inf _{y \in B} \rho(x, y), \sup _{x \in B} \inf _{y \in A} \rho(x, y)\right\}
$$

или метрика Помпейю [2, гл. 4, п. 4.5.22, замечание, с. 441]:

$$
\operatorname{dist}_{1}(A, B)=\frac{1}{2}\left(\sup _{x \in A} \inf _{y \in B} \rho(x, y)+\sup _{x \in B} \inf _{y \in A} \rho(x, y)\right) .
$$

Ответ на вопрос, элементарна ли любая изометрия $\exp X$ на себя, существенно зависит от структуры базового пространства. Непосредственно проверяется, что для

Работа выполнена при поддержке программы “Университеты России”, грант № УР.04.01.050. 
$X=\left\{a_{1}, a_{2}, a_{3}\right\} \subset \mathbb{R}^{1}, a_{1}<a_{2}<a_{3}$, отображение $F: \exp X \rightarrow \exp X$, переставляющее $\left\{a_{1}, a_{2}, a_{3}\right\}$ с $\left\{a_{1}, a_{3}\right\}$ и тождественное на остальных элементах, является изометрией относительно расстояния dist, но не порождается изометрией пространства $X$.

Для гиперпространства $\exp \mathbb{R}^{n}$ всех непустых компактных подмножеств $\mathbb{R}^{n}$ с хаусдорфовой метрикой dist любая его изометрия на себя является элементарной [5], [6, c. 170]. Аналогичньй результат имеется для гиперпространства $\exp \mathbb{T}^{n}$ над тором $\mathbb{T}^{n}=$ $\mathbb{R}^{n} / \mathbb{Z}^{n}$ с обычной метрикой $\rho(x, y)=\inf \left\{\|x-y+l\|: l \in \mathbb{Z}^{n}\right\}$ на $\mathbb{T}^{n}$ (см. [7, теорема 3 , c. 122]), а также для гиперпространства $\exp S^{n}$ над сқерой $S^{n}$ в $(n+1)$-мерном строго выпуклом гладком нормированном пространстве (см. [7, теорема 4, с. 123]). И наконец, элементарность изометрий гиперпространства $\exp X$ доказана для конечномерных нормированных пространств $X$ со строго вьпуклой нормой и для римановых многообразий $X$ (без края) с внутренней метрикой (в частности, для любой выпуклой области $\left.X \subset \mathbb{R}^{n}\right)[8$, теорема 4 , с. 181$]$.

В этой статье в качестве базового пространства мы рассматриваем произвольное неодноточечное компактное множество $K \subset \mathbb{R}^{1}$, и для гиперпространства $\exp K$, наделенного обобщенной метрикой Помпейю

$$
\operatorname{dist}_{\alpha}(A, B)=\frac{1}{2^{1 / \alpha}}\left[\left(\sup _{x \in A} \inf _{y \in B}|x-y|\right)^{\alpha}+\left(\sup _{x \in B} \inf _{y \in A}|x-y|\right)^{\alpha}\right]^{1 / \alpha}
$$

с $1<\alpha<+\infty$, введенной в п. 2 , доказьваем в п. 3 элементарность любой его изометрии на себя. Пример с трехточечньм множеством показывает, что это утверждение перестает быть верным при использовании классической метрики Хаусдорфа (1), и нам неизвестно, верно ли оно для гиперпространств, наделенных метрикой Помпейю (2), т.е. при $\alpha=1$. Естественно, остается открытым и вопрос об аналоге основной теоремы для произвольных компактных множеств в $K \subset \mathbb{R}^{n}$ при $n>1$.

Всюду в тексте под изометрией $g: X \rightarrow Y$ подразумевается изометрическое отображение $X$ на $Y$, в случае же изометрического отображения в мы говорим об изометрическом вложсени. Тождественное отображение обозначено символом Id. Для отображения $f: X \rightarrow Y$ символом $\exp f$ обозначается отображение $\exp X \rightarrow \exp Y$, определяемое формулой $(\exp f)(A)=f(A)=\{f(x): x \in A\}$. Пустое множество обозначено символом $\varnothing$. Без специальных оговорок используются следующие известные факты: изометрическое вложение компактного метрического пространства в себя всегда сюръективно, т.е. является изометрией $[9$, гл. $3, \S 3$, упражнение 30, с. 251]); для компактного метрического пространства $X$ гиперпространство $\exp X$ компактно [1, т. 2 , $\S 42$, теорема 1 , с. 52$]$.

2. Модификация метрики Помпейю. Следуя [10, с. 479], мы называем квазиметрикой функцию расстояния, удовлетворяющую всем аксиомам метрики, кроме аксиомы симметричности. Отметим, что любое билипшицево отображение квазиметрических пространств инъективно (см. [11, теорема 8, с. 136]). Для квазиметрики $\sigma$ функция $\sigma^{*}(x, y):=\sigma(y, x)$ задает квазиметрику, сопряженную к $\sigma[12$, определение 2.2 , с. 72]. Пара взаимно сопряженных квазиметрик $\sigma$ и $\sigma^{*}$ естественно порождает метрику на $X$, если, к примеру, положить $d(x, y)=\max \left\{\sigma(x, y), \sigma^{*}(x, y)\right\}$ или $d(x, y)=\sigma(x, y)+\sigma^{*}(x, y)($ см. $[12$, c. 84-85]).

Имеется общий способ перехода от квазиметрики к метрике в пространстве $X$. (Заметим, что нас не интересует топология, порожденная на $X$ исходной квазиметрикой, и мы 
не решаем задачу метризации пространства $X$ в этой топологии. По поводу квазиметризуемости топологических пространств или метризуемости квазиметрических пространств см., например, [12, теорема 2.8, с. 75] или $[13, \S 2$, пп. 2.2-2.5]). В качестве симметризатора рассматривается вешественная неотрицательная функция $P:[0,+\infty) \times$ $[0,+\infty) \rightarrow \mathbb{R}^{1}$, удовлетворяющая условиям

1) $P(a, b)=0$ тогда и только тогда, когда $a=b=0$;

2) $P(a, b)=P(b, a)$;

3) $P\left(a_{1}+a_{2}, b_{1}+b_{2}\right) \leqslant P\left(a_{1}, b_{1}\right)+P\left(a_{2}, b_{2}\right)$;

4) если $a_{1}<a_{2}$ и $b_{1} \leqslant b_{2}$, то $P\left(a_{1}, b_{1}\right)<P\left(a_{2}, b_{2}\right)$.

Если при этом

5) $P(t, t)=t$ для всех $t \geqslant 0$,

то $P$ назьваем допустимым симметризатором.

УТВЕРЖДЕНИЕ 2.1. Для любой квазиметрики $\sigma$ на множестве $X$ и для любого симметризатора $Р$ функиия

$$
(P \circ \sigma)(x, y):=P\left(\sigma(x, y), \sigma^{*}(x, y)\right)
$$

является метрикой на X.

ДокАЗАТЕЛЬСтво. Справедливость аксиом тождества и симметрии для $P$ о $\sigma$ очевидна. Для $x, y, z \in X$, используя аксиому треугольника для квазиметрик $\sigma$ и $\sigma^{*}$, свойство монотонности 4) и свойство полуаддитивности 3 ) симметризатора $P$, получаем неравенство треугольника:

$$
\begin{aligned}
(P \circ \sigma)(x, z) & =P\left(\sigma(x, z), \sigma^{*}(x, z)\right) \leqslant P\left(\sigma(x, y)+\sigma(y, z), \sigma^{*}(x, y)+\sigma^{*}(y, z)\right) \\
& \leqslant P\left(\sigma(x, y), \sigma^{*}(x, y)\right)+P\left(\sigma(y, z), \sigma^{*}(y, z)\right)=(P \circ \sigma)(x, y)+(P \circ \sigma)(y, z) .
\end{aligned}
$$

Утверждение доказано.

В $[10, \S 5$, с. 482$]$ отмечено, что для метрического пространства $(X, \rho)$ формула

$$
\sigma(A, B)=\sup _{x \in A} \inf _{y \in B} \rho(x, y)
$$

задает квазиметрику в гиперпространстве $\exp X$. За ней мы в дальнейшем тексте закрепим обозначение $\sigma$. Используя симметризатор

$$
P_{\alpha}(a, b)=\frac{1}{2^{1 / \alpha}}\left(a^{\alpha}+b^{\alpha}\right)^{1 / \alpha} \quad \text { при } \quad 1 \leqslant \alpha<+\infty,
$$

получаем в силу 2.1 метрику $\operatorname{dist}_{\alpha}=P_{\alpha} \circ \sigma$ на $\exp X$, которую в случае $\alpha>1$ назовем обобщенной метрикой Помпейю. (Функция $P_{\infty}(a, b)=\max \{a, b\}$ не удовлетворяет условию 4) строгой монотонности, и поэтому классическая метрика Хаусдорфа (1) выпадает из дальнейших рассмотрений.)

УТВЕРЖДЕНИЕ 2.2. Пусть гиперпространства $\exp X$ и $\exp Y$ над компактными метрическими пространствами $\left(X, \rho_{1}\right)$ и $\left(Y, \rho_{2}\right)$ наделены метриками Роб при одном и том же симметризаторе $P$, и пусть имеется изометрия $F: \exp X \rightarrow \exp Y$. Если $A, B \in \exp X$ таковы, что $A \subset B$ и для некоторой точки $b \in F(B)$ выполнено неравенство $\inf _{x \in F(A)} \rho_{2}(x, b) \geqslant \sigma(B, A)$, mo $F(A) \subset F(B)$. 
ДоКАЗАТЕЛЬСТВо. Если $a \in F(A) \backslash F(B)$, то

$$
\sigma(F(A), F(B)) \geqslant \inf _{y \in F(B)} \rho_{2}(a, y)>0 .
$$

Tak как

$$
P(0, \sigma(F(B), F(A))<(P \circ \sigma)(F(A), F(B))=(P \circ \sigma)(A, B)=P(0, \sigma(B, A)),
$$

то строгая монотонность 4) симметризатора $P$ приводит к противоречию:

$\sigma(F(B), F(A))<\sigma(B, A) \leqslant \inf _{x \in F(A)} \rho_{2}(x, b) \leqslant \sup _{y \in F(B)} \inf _{x \in F(A)} \rho_{2}(x, y)=\sigma(F(B), F(A))$.

Следовательно, $F(A) \backslash F(B)=\varnothing$, и утверждение доказано.

Следуя [7, с. 118], пару точек $x, y$ компактного метрического пространства $(X, \rho)$ назьваем антиподальной парой, если $\rho(x, y)=\operatorname{diam} X$; при этом точки $x$ и $y$ являются взаимньми антиподами. Метрическое пространство, в котором каждая точка имеет не более одного антипода, назовем слабо-антиподальным пространством. (В определении [7, с. 118] антиподального пространства требовалось, чтобы у каждой точки был в точности один антипод). Очевидно, что изометрия компактных метрических пространств переводит антиподальную пару точек в антиподальную и сохраняет свойство слабой антиподальности. Усиленным аналогом $[7$, формула (3), с. 118] является следующее

УТВЕРЖДЕНИЕ 2.3. Пусть $(X, \rho)$ - компактное метрическое пространство, $P$ - допустимый симметризатор. Гиперпространство $\exp X$ с метрикой $P \circ \sigma$ слабо-антиподально в точности тогда, когда слабо-антиподально базовое пространство $X$. Множсество всех антиподальных пар в $\exp X$ совпадает с парами одноточечных мнохсеств $\{a\},\{b\}$, соответствующ, антиподальным парам $a, b \in X$.

ДоказАтельство. Пусть $d:=\operatorname{diam} X$. Так как $\sigma(A, B) \leqslant d$ для всех $A, B \in \exp X$, то $(P \circ \sigma)(A, B) \leqslant P(d, d)=d$. В силу компактности имеется антиподальная пара $a, b \in X$, и

$$
(P \circ \sigma)(\{a\},\{b\})=P(\rho(a, b), \rho(b, a))=P(d, d)=d .
$$

Следовательно, $\operatorname{diam} \exp X=d$. Если $\rho\left(a_{1}, b_{1}\right)=d$, то $(P \circ \sigma)\left(\left\{a_{1}\right\},\left\{b_{1}\right\}\right)=P(d, d)=d$, и $\left\{a_{1}\right\},\left\{b_{1}\right\}$ - антиподальная пара в $\exp X$.

Пусть слабо-антиподально пространство $\exp X$. Тогда из антиподальности пар $a$, $b \in X$ и $a, c \in X$ следует антиподальность пар $\{a\},\{b\}$ и $\{a\},\{c\}$ в $\exp X$, а из слабой антиподальности $\exp X$ следует равенство $\{c\}=\{b\}$, т.е. $c=b$, и мы получаем слабую антиподальность для $X$.

Пусть теперь слабо-антиподально пространство $X$. Если $(P \circ \sigma)(A, B)=d$, то в силу строгой монотонности и допустимости симметризатора $P$ вьполнено $\sigma(A, B)=$ $\sigma(B, A)=d$. В силу компактности существуют точки $a \in A$ и $b \in B$, для которых $d=\inf _{y \in B} \rho(a, y)$ и $d=\inf _{x \in A} \rho(x, b)$, откуда следует антиподальность в $X$ любой пары $a, y$ для $y \in B$ и любой пары $x, b$ для $x \in A$. В силу слабой антиподальности пространства $X$ это возможно лишш в случае, когда $B=\{b\}$ и $A=\{a\}$. Для двух 
антиподальных пар $A, B$ и $A, C$ в $\exp X$ имеем $A=\{a\}, B=\{b\}, C=\{c\}$, где пары $a$, $b$ и $a, c$ антиподальны в $X$. Следовательно, $b=c$ и поэтому $B=C$, что и доказьвает слабую антиподальность $\exp X$. Утверждение доказано.

Компактное слабо-антиподальное метрическое пространство $(X, \rho)$ назьваем $\partial$-антиподальным, если для любого $x \in X$ существует такая антиподальная пара $a, b \in X$, что $\rho(a, b)=\rho(a, x)+\rho(x, b)$.

Теорема 2.4. Пусть $X, Y$ - компактные множества в $\mathbb{R}^{n}$, рассматриваемые как метрические пространства с обычным евклидовым расстоянием. Пусть гиперпространства $\exp X, \exp Y$ наделены обобщенной метрикой Помпейю $\operatorname{dist}_{\alpha}=$ $P_{\alpha} \circ \sigma$ (см. (3)). Если мнохсество $X$ является д-антиподальным, то для любой изометрии $F: \exp X \rightarrow \exp Y$ существует изометрия $f: X \rightarrow Y$ такая, что $F(\{x\})=\{f(x)\}$ для всех $x \in X$.

ДокАЗАТЕЛЬСтво. Пусть $d:=\operatorname{diam} X, x \in X$ и $a, b$ - такая антиподальная пара в $X$, что $|a-x|+|x-b|=d$. Пусть $F(\{x\})=Z$. По утверждению 2.3 вьполнены равенства $F(\{a\})=\left\{a^{\prime}\right\}$ и $F(\{b\})=\left\{b^{\prime}\right\}$, где $a^{\prime}, b^{\prime}$ - антиподальная пара в $Y$, т.е. $\left|a^{\prime}-b^{\prime}\right|=d$. Поэтому при $x=a$ или $x=b$ множество $Z$ является одноточечньг. Пусть теперь $x \neq a, b$ и, следовательно, $0<|a-x|<d, 0<|x-b|<d$. Из изометричности $F$ следует, что $0<\operatorname{dist}_{\alpha}\left(\left\{a^{\prime}\right\}, Z\right)<d$ и $0<\operatorname{dist}_{\alpha}\left(\left\{b^{\prime}\right\}, Z\right)<d$. В частности, для всех $z \in Z$ вьполняются неравенства $\left|z-a^{\prime}\right|<d$ и $\left|z-b^{\prime}\right|<d$. Поэтому ортогональная проекция $Z^{\prime}$ множества $Z$ на прямую, проходящую через точки $a^{\prime}$ и $b^{\prime}$, лежит строго внутри отрезка $\left[a^{\prime} b^{\prime}\right]$. Пусть отрезок $\left[c_{1} c_{2}\right]$ является выпуклой оболочкой множества $Z^{\prime}$ и $d=s_{1}+l+s_{2}$, где $s_{1}=\left|c_{1}-a^{\prime}\right|, l=\left|c_{2}-c_{1}\right|, s_{2}=\left|c_{2}-b^{\prime}\right|$. Заметив, что квазирасстояние $\sigma$ между множествами не увеличивается при их проекции на прямую, а затем воспользовавшись монотонностью симметризатора $P_{\alpha}$ и его полуаддитивностью 3 ), получаем длинное соотношение

$$
\begin{aligned}
d \geqslant\left(P_{\alpha} \circ \sigma\right)\left(\left\{a^{\prime}\right\}, Z^{\prime}\right)+\left(P_{\alpha} \circ \sigma\right)\left(\left\{b^{\prime}\right\}, Z^{\prime}\right) & =P_{\alpha}\left(s_{1}, s_{1}+l\right)+P_{\alpha}\left(s_{2}+l, s_{2}\right) \\
& \geqslant P_{\alpha}\left(s_{1}+s_{2}+l, s_{1}+l+s_{2}\right)=P_{\alpha}(d, d)=d
\end{aligned}
$$

из которого следует, что

$$
\left[\left(s_{1}\right)^{\alpha}+\left(s_{1}+l\right)^{\alpha}\right]^{1 / \alpha}+\left[\left(s_{2}+l\right)^{\alpha}+\left(s_{2}\right)^{\alpha}\right]^{1 / \alpha}=\left[d^{\alpha}+d^{\alpha}\right]^{1 / \alpha} .
$$

В двумерном векторном пространстве, наделенном нормой $\|\{x, y\}\|=\left(|x|^{\alpha}+|y|^{\alpha}\right)^{1 / \alpha}$, где $\alpha>1$, неравенство треугольника представляется частньм случаем неравенства Минковского:

$$
\left(\left|x_{1}+x_{2}\right|^{\alpha}+\left|y_{1}+y_{2}\right|^{\alpha}\right)^{1 / \alpha} \leqslant\left(\left|x_{1}\right|^{\alpha}+\left|y_{1}\right|^{\alpha}\right)^{1 / \alpha}+\left(\left|x_{2}\right|^{\alpha}+\left|y_{2}\right|^{\alpha}\right)^{1 / \alpha},
$$

равенство в котором реализуется только в случае коллинеарности векторов $\left\{x_{1}, y_{1}\right\}$ и $\left\{x_{2}, y_{2}\right\}$ (см., например, $[14$, c. 16]). Усмотрев в длинном соотношении случай равенства в неравенстве (6) для двумерных векторов $\left\{s_{1}, s_{1}+l\right\}$ и $\left\{s_{2}+l, s_{2}\right\}$, мы получаем условие коллинеарности этих векторов, т.е. равенства $s_{1}=k\left(s_{2}+l\right)$ и $s_{1}+l=k s_{2}$ с некоторым $k \in \mathbb{R}^{1}$. Но эти равенства возможны лишш при $l=0$. Следовательно, $c_{1}=c_{2}=z^{\prime}$ и $Z^{\prime}=\left\{z^{\prime}\right\}$. Из того же длинного соотношения следует и равенство

$$
\left(P_{\alpha} \circ \sigma\right)\left(\left\{a^{\prime}\right\}, Z\right)=\left(P_{\alpha} \circ \sigma\right)\left(\left\{a^{\prime}\right\},\left\{z^{\prime}\right\}\right)=\left|z^{\prime}-a^{\prime}\right|
$$


Если $y \in Z \backslash\left\{z^{\prime}\right\}$, то $\sigma\left(Z,\left\{a^{\prime}\right\}\right) \geqslant\left|y-a^{\prime}\right|>\left|z^{\prime}-a^{\prime}\right|$ и $\sigma\left(\left\{a^{\prime}\right\}, Z\right) \geqslant\left|z^{\prime}-a^{\prime}\right|$, и поэтому $\left(P_{\alpha} \circ \sigma\right)\left(\left\{a^{\prime}\right\}, Z\right)>P_{\alpha}\left(\left|z^{\prime}-a^{\prime}\right|,\left|z^{\prime}-a^{\prime}\right|\right)=\left|z^{\prime}-a^{\prime}\right|$, что противоречит (7). Поэтому $Z \backslash\left\{z^{\prime}\right\}=\varnothing$ и $Z=\left\{z^{\prime}\right\}$.

Тем самьм доказано, что $F(\{x\})$ - одноточечное множество при любом $x \in X$. Поэтому отображение $f(x)=y$, где $\{y\}=F(\{x\})$, корректно определено и дает требуемую изометрию $X$ на $Y$. Теорема доказана.

Итак, в классе $\partial$-антиподальных компактов $X \subset \mathbb{R}^{n}$ при использовании обобщенной метрики Помпейю (3) проблема элементарности изометрий гиперпространств сводится к вопросу о тривиальности любой изометрии $F: \exp X \rightarrow \exp X$, тождественной на подпространстве одноточечных множеств. Доказательство теоремы 2.4 не проходит при $\alpha=1$ и поэтому метрика Помпейю (2), к сожалению, тоже выпадает из дальнейшего рассмотрения.

3. Случай компактных подмножеств на прямой. Так как любое компактное неодноточечное множество $K \subset \mathbb{R}^{1}$ является $\partial$-антиподальным, то при использовании обобщенной метрики Помпейю (3) описание изометрий гиперпространств $\exp K_{1} \rightarrow$ $\exp K_{2}$ над компактами $K_{1}, K_{2} \subset \mathbb{R}^{1}$ сводится теоремой 2.4 к вопросу о тривиальности изометрий $F: \exp K_{1} \rightarrow \exp K_{1}$, тождественных на подпространстве одноточечных множеств, ответ на которьй будет дан теоремой 3.3. Далее (по умолчанию) предполагается, что гиперпространство $\exp K$ наделено обобщенной метрикой Помпейю dist $_{\alpha}=$ $P_{\alpha} \circ \sigma$. Для компактных множеств $A \subset \mathbb{R}^{1}$ используем обозначения $\min A=\min \{x:$ $x \in A\}$ и $\max A=\max \{x: x \in A\}$.

Лемма 3.1. Пусть $K \subset \mathbb{R}^{1}$ - неодноточечное компактное мнохсество и изометрия $F: \exp K \rightarrow \exp K$ тохдественна на одноточечных мнохествах. Тогда $\min F(A)=\min A u \max F(A)=\max A$ для всеx $A \in \exp K$.

ДокАЗАТЕЛЬСТво. Пусть $a=\min K, b=\max K, x_{0}=\min A, x_{1}=\max A, l=$ $\left|x_{1}-x_{0}\right|, y_{0}=\min F(A), y_{1}=\max F(A)$. Из равенств

$$
\operatorname{dist}_{\alpha}\left(F(A),\left\{x_{0}\right\}\right)=\operatorname{dist}_{\alpha}\left(A,\left\{x_{0}\right\}\right)=P_{\alpha}\left(\left|x_{1}-x_{0}\right|, 0\right)=2^{-1 / \alpha} l
$$

следует, что $\sigma\left(F(A),\left\{x_{0}\right\}\right) \leqslant l$ и $F(A) \subset\left[x_{0}-l, x_{1}\right]$. Аналогично, сравнив расстояния от $F(A)$ и $A$ до $\left\{x_{1}\right\}$, получим включение $F(A) \subset\left[x_{0}, x_{1}+l\right]$, которое вместе с предыдущим дает оценки $x_{0} \leqslant y_{0}, y_{1} \leqslant x_{1}$. Применив то же рассуждение к обратной изометрии $F^{-1}$ и компакту $F(A)$, получим включение $A \subset\left[y_{0}, y_{1}\right]$ и неравенства $y_{0} \leqslant x_{0}, x_{1} \leqslant y_{1}$, которые вместе с предыдущими оценками приводят к равенствам $x_{0}=y_{0}, x_{1}=y_{1}$. Лемма доказана.

Лемма 3.2. Пусть $K \subset \mathbb{R}^{1}$ - неодноточечное компактное множсество и изометрия $F: \exp K \rightarrow \exp K$ тохдественна на одноточечных мнохсествах. Тогда $F(A)=A$ для любого конечного мнохества $A \subset K$.

ДокАЗАТЕЛЬСтво проведем индукцией по $n=\operatorname{card} A$. Для $n=1$ заключение леммы тривиально. Пусть известно, что для любого неодноточечного компакта $K \subset \mathbb{R}^{1}$, любой изометрии $F: \exp K \rightarrow \exp K$, тождественной на одноточечных множествах, и любого множества $A \subset K$ c card $A \leqslant n$ вьполняется равенство $F(A)=A$.

1) Докажем, что для любого неодноточечного компакта $K \subset \mathbb{R}^{1}$, любой изометрии $F: \exp K \rightarrow \exp K$, тождественной на одноточечных множествах, и любого $A \subset K$ с 
$\operatorname{card} A \leqslant(n+1)$ справедливо включение $F(A) \supset A$. Пусть $A=\left\{a_{0}, \ldots, a_{n}\right\} \subset K$ и $a_{0}<\cdots<a_{n}$. Для $B=A \backslash\left\{a_{n}\right\}$ по предположению индукции имеем равенство $F(B)=B$. Лемма 3.1 дает равенства $a_{0}=\min A=\min F(A), a_{n}=\max A=\max F(A)$, из которых следует, что $a_{0}, a_{n} \in F(A)$. К включению $B \subset A$ и точке $a_{n} \in F(A)$, для которой

$$
\inf _{x \in F(B)=B}\left|x-a_{n}\right|=\left|a_{n}-a_{n-1}\right| \geqslant \sigma(A, B)=\left|a_{n}-a_{n-1}\right|,
$$

применяем утверждение 2.2 и получаем включение $B=F(B) \subset F(A)$, из которого следует, что $A=B \cup\left\{a_{n}\right\} \subset F(A)$.

2) Докажем, что для любого неодноточечного компакта $K \subset \mathbb{R}^{1}$, любой изометрии $F: \exp K \rightarrow \exp K$, тождественной на одноточечных множествах, и любого $A \subset K$ с $\operatorname{card} A \leqslant(n+1)$ точка $\max F(A)$ является изолированной в $F(A)$. Допустим противное. Тогда для некоторых $K, F, A=\left\{a_{0}, \ldots, a_{n}\right\} \subset K$, где $a_{0}<\cdots<a_{n}$, найдется $h \in\left(a_{n-1}, a_{n}\right) \cap F(A)$. Положим $H=F(A) \cap\left[a_{0}, h\right]$ и $D=H \cup\left\{a_{n}\right\}$. К изометрии $F^{-1}$, включению $H \subset F(A)$ и точке $a_{n} \in A=F^{-1}(F(A))$, для которой

$$
\begin{aligned}
\inf _{x \in F^{-1}(H)}\left|a_{n}-x\right| & =\left|a_{n}-\max F^{-1}(H)\right|=\left|a_{n}-\max H\right|=\left|a_{n}-h\right| \\
& \geqslant \sigma(F(A), H)=\left|a_{n}-h\right|,
\end{aligned}
$$

применяем утверждение 2.2 и получаем включение $F^{-1}(H) \subset A$. Так как $a_{n} \notin F^{-1}(H)$ $\subset\left[a_{0}, h\right]$, то $F^{-1}(H) \subset\left\{a_{0}, \ldots, a_{n-1}\right\}$, что тотчас приводит к противоречию: $h=$ $\max H=\max F^{-1}(H) \leqslant a_{n-1}$. Следовательно, точка $a_{n}$ не может быть предельной для $F(A)$.

3) Наконец, докажем, что для любого неодноточечного компакта $K \subset \mathbb{R}^{1}$, любой изометрии $F: \exp K \rightarrow \exp K$, тождественной на одноточечных множествах, и любого множества $A \subset K$ c card $A \leqslant(n+1)$ вьполняется равенство $F(A)=A$. Пусть $A=\left\{a_{0}, \ldots, a_{n}\right\} \subset K$ и $a_{0}<\cdots<a_{n}$. Так как на этапе 2) установлено, что точка $a_{n}=\max F(A)$ изолирована в $F(A)$, то множество $C=F(A) \backslash\left\{a_{n}\right\}$ компактно. Положим $c=\max C$ и, применив лемму 3.1 к изометрии $F^{-1}$, получим равенства $\min F^{-1}(C)=\min C=a_{0}, \max F^{-1}(C)=\max C=c<a_{n}$. К изометрии $F^{-1}$, включению $C \subset F(A)$ и точке $a_{n} \in A=F^{-1}(F(A))$, для которой

$$
\inf _{x \in F^{-1}(C)}\left|a_{n}-x\right|=\left|a_{n}-c\right| \geqslant \sigma\left(A, F^{-1}(C)\right)=\left|a_{n}-c\right|,
$$

применяем утверждение 2.2 и получаем включение $F^{-1}(C) \subset A$, из которого следует, что множество $F^{-1}(C) \subset\left\{a_{0}, \ldots, a_{n-1}\right\}$ имеет не более $n$ точек. По предположению индукции $C=F\left(F^{-1}(C)\right)=F^{-1}(C)$ и, следовательно, $C \subset\left\{a_{0}, \ldots, a_{n-1}\right\}$. Поэтому $F(A)=C \cup\left\{a_{n}\right\} \subset A$. Вместе с включением $F(A) \supset A$, доказанньг на первом этапе, это приводит к требуемому равенству $F(A)=A$. Лемма доказана.

Теорема 3.3. Пусть $K_{1}, K_{2}$ - неодноточечные компактные мнохества в $\mathbb{R}^{1}$, гиперпространства над которыми наделены обобщенной метрикой Помпейю $\operatorname{dist}_{\alpha}$. Тогда любая изометрия $F: \exp K_{1} \rightarrow \exp K_{2}$ әлементарна, m.e. $F=\exp f$ для некоторой изометрии $f: K_{1} \rightarrow K_{2}$. 
ДокАЗАтЕЛЬСтво. Теорема 2.4 дает изометрию $f: K_{1} \rightarrow K_{2}$ такую, что $F(\{x\})=$ $\{f(x)\}$ для всех $x \in K_{1}$. Отображение $\exp f: \exp K_{1} \rightarrow \exp K_{2}$ является изометрией, совпадающей с $F$ на подпространстве одноточечных множеств. Следовательно, изометрия $G=(\exp f)^{-1} \circ F: \exp K_{1} \rightarrow \exp K_{1}$ тождественна на одноточечных множествах. В силу леммы 3.2 изометрия $G$ тождественна на подпространстве $\mathscr{P}$ всех конечных подмножеств компакта $K_{1}$. В силу непрерьвности изометрии $G$ и плотности подпростран-

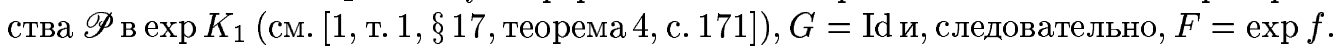
Теорема доказана.

Вопрос об изометрических вложениях $F: \exp K_{1} \rightarrow \exp K_{2}$ представляется более сложным, как показьвает следующий пример. Пусть $K_{1}=[0,1], K_{2}=[0,4]$ и $F$ : $\exp K_{1} \rightarrow \exp K_{2}$ задано формулой $F(A)=A \cup T$, где $T$ - фиксированньй компакт $T \subset[3,4]$. Легко убедиться, что $F$ - изометрическое вложение, но оно не порождается никаким изометрическим вложением $f: K_{1} \rightarrow K_{2}$.

Полное описание изометрий гиперпространства над произвольньм компактным множеством в $\mathbb{R}^{1}$ позволяет получить теоремы устойчивости для квазиизометрических вложений в гиперпространствах, наделенных обобшенной метрикой Помпейю, которые имеют естественные приложения в теории многозначных функций и селекторов (см. $[1$, т. 2 , гл. $4, \S 43$, с. 82$]$ ), а также в некоторых задачах интервального анализа.

Авторы глубоко признательны рецензенту за сделанные им замечания, которые способствовали значительному улучшению текста статьи.

\section{СПИСОК ЦИТИРОВАННОЙ ЛИТЕРАТУРЫ}

[1] Куратовский К. Топология. Т. 1, 2. М.: Мир, 1966, 1969.

[2] Энгелькинг Р. Общая топология. М.: Мир, 1986.

[3] Заричный М. М., Федорчук В. В.Ковариантные функторы в категориях топологических пространств // Итоги науки и техники. Алгебра. Топология. Геометрия. Т. 28. М.: ВИНИТИ, 1990. С. 47-95.

[4] Michael E. Topologies on spaces of subsets // Trans. Amer. Math. Soc. 1951. V. 51. № 1. P. 152-182.

[5] Gruber P. M. The space of compact subsets of $E^{d} / /$ Geom. Dedicata. 1979. V. 8. P. 87-90.

[6] Gruber P. M., Letti G. Isometries of the space of compact subsets of $E^{d} / /$ Stud. Sci. Math. Hungar. 1979. V. 14. P. 169-181.

[7] Gruber P. M., Tichy R. Isometries of spaces of compact or compact convex subsets of metric manifolds // Monat. Math. 1982. V. 93. № 2. P. 117-126.

[8] Bandt Ch. On the metric structure of hyperspaces with Hausdorff metric // Math. Nachr. 1986. V. 129. P. $175-183$.

[9] Александрян Р. А., Мирзаханян Э. А. Общая топология. М.: Высшая школа, 1979.

[10] Albert G.E. A note on quasi-metric spaces // Bull. Amer. Math. Soc. 1941. V. 47. №6. P. 479-482.

[11] Reilly I. L., Subrahmanyan P. V., Vamanamurthy M.K. Cauchy sequences in quasi-pseudo-metric spaces // Monat. Math. 1982. V. 93. № 2. P. 127-140.

[12] Kelly J. C. Bitopological spaces // Proc. London. Math. Soc. 1963. V. 13. P. 71-83.

[13] Sion M., Zelmer G. On quasi-metrizability // Canad. J. Math. 1967. V. 19. №6. P. 1243-1249.

[14] Соболев С. Л. Некоторые применения функционального анализа в математической физике. 3-е изд. М.: Наука, 1988.

(В.В. Асеев) Институт математики СО РАН 